9th A. Friedmann International Seminar and

3rd Casimir Symposium 2015

International Journal of Modern Physics: Conference Series

Vol. 41 (2016) 1660125 (6 pages)

(C) The Author(s)

DOI: $10.1142 /$ S2010194516601253

\title{
Hypermotion due to space-time deformation
}

\author{
Michael Fil'chenkov* and Yuri Laptev ${ }^{\dagger}$ \\ Institute of Gravitation and Cosmology, \\ Peoples' Friendship University of Russia, \\ 6 Miklukho-Maklay Street \\ Moscow, Russia 117198, Russia \\ *fmichael@mail \\ †yplaptev@rambler.ru
}

Received 4 September 2015

Revised 4 November 2015

Published 18 March 2016

\begin{abstract}
A superluminal motion (hypermotion) via M. Alcubierre's warp drive is considered. Parameters of the warp drive have been estimated. The equations of starship geodesics have been solved. The starship velocity have been shown to exceed the speed of light, with the local velocity relative to the deformed space-time being below it. Hawking's radiation does not prove to affect the ship interior considerably. Difficulties related to a practical realization of the hypermotion are indicated.
\end{abstract}

Keywords: warp drive; geodesics; Hawking radiation.

PACS numbers: 0420, 0490

\section{Introduction}

If one assumes that our Galaxy has been explored by developed extraterrestrial civilizations in the same way as we had been sailing the world's ocean in the Age of Exploration and the Great Navigations, it is necessary for different civilizations to traverse the distances that separate them.

Mankind created various means to traverse distances, but it is the rockets that allowed us to escape the Earth and explore the Solar system. The external boundary of the Solar system is 0.6 pc. Proxima Centauri, star nearest to the Sun, is 1.3 distant. The Galaxy radius is $15 \mathrm{kpc}$. The Sun is located at a distance of $8 \mathrm{kpc}$ from the Galaxy center.

Hence, it is clear that exploring the Galaxy is practically impossible in human lifetime. While moving at a velocity close to the speed of light (via a photon engine),

This is an Open Access article published by World Scientific Publishing Company. It is distributed under the terms of the Creative Commons Attribution 4.0 (CC-BY) License. Further distribution of this work is permitted, provided the original work is properly cited. 
any distances are reachable due to relativistic time dilation, but it is a voyage without return, since on Earth hundreds of thousand years will elapse.

Here we arrive at a conclusion that the Galaxy tour would be possible, only while moving at a superluminal speed (hypermotion) relative to the start point, with a local velocity not exceeding the speed of light. The only possibility lies in diminishing the distance to be traversed by a starship as compared with its separation from the destination point at the instant of starship start. A wormhole connecting the start and destination points through a handle is one of the possibilities of coming to the destination point using a shorter route. Another possibility is to contract the spacetime ahead of the starship. This process is performed with the aid of the so-called warp drive proposed by Miguel Alcubierre in 1994, ${ }^{1}$ although the idea of the warp drive appeared earlier in science fiction. A lot of papers have been published since then (see, e.g.,Refs. 2 and 3). Some of them ${ }^{3}$ contain only numerical calculations.

Our paper is aimed at obtaining only analytical results in the framework of a one-dimensional model.

\section{Metric, Energy Density, Alcubierre Bubble Sizes}

The distorted space-time is described by the metric

$$
d s^{2}=c^{2} d t^{2}-\left[d x-v_{s} f\left(r_{s}\right) d t\right]^{2}-d y^{2}-d z^{2},
$$

where

$$
\begin{gathered}
r_{s}^{2}=\left(x-x_{s}\right)^{2}+y^{2}+z^{2}, \\
v_{s}=\frac{d x_{s}}{d t}, \\
f\left(r_{s}\right)=\frac{\tanh \sigma\left(r_{s}+R\right)-\tanh \sigma\left(r_{s}-R\right)}{2 \tanh \sigma R},
\end{gathered}
$$

where $0 \leq f \leq 1$ ( $f=0$ for $r_{s} \gg R, f=\frac{1}{2}$ at $r_{s}=R, f=1$ at $\left.r_{s}=0\right)$, $v_{s}$ is the starship velocity directed along an $x$-axis, i.e. $v_{y}=v_{z}=0$. Thus the problem is naturally one-dimensional.

A bubble of the radius $R$ and the wall thickness $\frac{1}{\sigma}$ has been created around the starship. Inside and outside the bubble the space-time is flat, and in the wall space-time is contracted in a forward direction and expanded in an opposite one. Thus, the starship brings the place of destination closer due to contraction of the space-time ahead of it.

Alcubierre's bubble as well as the wormhole is supported by a negative energy. The energy density required to support the bubble

$$
\varepsilon=-\frac{c^{2} v_{s}^{2} \sigma^{2} \rho^{2}}{128 \pi G r_{s}^{2}},
$$

where $\rho^{2}=y^{2}+z^{2}=$ const, $r_{s}=\rho=$ const at $x=x_{s}$. 
The volume of the layer with nonzero energy density

$$
V=\frac{4 \pi R^{2}}{\sigma}
$$

The effective mass

$$
M=\frac{|\varepsilon| V}{c^{2}}=\frac{v_{s}^{2} \rho^{2} \sigma R^{2}}{32 G r_{s}^{2}} .
$$

The gravitational radius

$$
r_{g}=\frac{2 G M}{c^{2}}=\frac{v_{s}^{2} \rho^{2} \sigma R^{2}}{16 c^{2} r_{s}^{2}}
$$

De Sitter's horizon

$$
r_{0}=\frac{c^{2}}{2} \sqrt{\frac{3}{2 \pi G|\varepsilon|}}=\frac{4 \sqrt{3} c r_{s}}{v_{s} \rho \sigma} .
$$

For $R>r_{g}>r_{0}$ we have restrictions on the bubble sizes as follows

$$
8 \cdot 3^{1 / 4}\left(\frac{c r_{s}}{v_{s} \rho}\right)^{3 / 2}<\sigma R<16\left(\frac{c r_{s}}{v_{s} \rho}\right)^{2},
$$

where

$$
\sigma R>3, \quad \frac{c r_{s}}{v_{s} \rho}>\frac{\sqrt{3}}{4} .
$$

Thus, the starship mass exceeds $10^{-2} M_{\odot}$, if $R>100 m$, where $M_{\odot}$ is the solar mass.

\section{Equation of Geodesics}

The equation of geodesics reads

$$
\Gamma_{k l}^{i} u^{k} u^{l}+\frac{d u^{i}}{d s}=0,
$$

where

$$
\Gamma_{k l}^{i}=\frac{g_{i m}}{2}\left(\frac{\partial g_{m k}}{\partial x^{l}}+\frac{\partial g_{m l}}{\partial x^{k}}-\frac{\partial g_{k l}}{\partial x^{m}}\right)
$$

are Christoffel symbols,

$$
u^{i}=\frac{d x^{i}}{d s}
$$

is the 4-velocity,

$$
\begin{gathered}
d s=c d t \sqrt{1-\frac{\left(v-v_{s} f\right)^{2}}{c^{2}}}, \\
v=\frac{d x}{d t}, \quad v_{s}=\frac{x_{s}}{t} .
\end{gathered}
$$

Here $v_{0}-v_{s} f$ is the local velocity of the starship relative to the deformed space-time not exceeding the speed of light of light, $v$ is the rate of deformation of space-time 
relative to Minkowski space at the point of starship start, $v_{s}$ is the starship velocity relative to its start point. Finally, the 4 -velocity takes the form

$$
u^{i}=\left(\frac{1}{\sqrt{1-\frac{\left(v-v_{s} f\right)^{2}}{c^{2}}}}, \frac{v}{c \sqrt{1-\frac{\left(v-v_{s} f\right)^{2}}{c^{2}}}}, 0,0\right) .
$$

In the one-dimensional case equation (12) reduces to a system of two equations

$$
\begin{aligned}
& \Gamma_{00}^{0} u^{0} u^{0}+2 \Gamma_{01}^{0} u^{0} u^{1}+\Gamma_{11}^{0} u^{1} u^{1}+\frac{d u^{0}}{d s}=0, \\
& \Gamma_{00}^{1} u^{0} u^{0}+2 \Gamma_{01}^{1} u^{0} u^{1}+\Gamma_{11}^{1} u^{1} u^{1}+\frac{d u^{1}}{d s}=0 .
\end{aligned}
$$

Assume that $f=$ const, which allows the geodesic equations to be solved analytically.

Christoffel's symbols take the form

$$
\begin{gathered}
\Gamma_{00}^{0}=-\frac{2 v_{s} \dot{v}_{s} f^{2}}{c^{3}}, \quad \Gamma_{00}^{1}=\left(\frac{2 v_{s}^{2} f^{2}}{c^{3}}-1\right) \frac{\dot{v}_{s} f}{c^{2}}, \quad \Gamma_{11}^{0}=\frac{\dot{v}_{s} f}{c^{2}}, \\
\Gamma_{01}^{0}=\Gamma_{01}^{1}=\Gamma_{11}^{1}=0 .
\end{gathered}
$$

Alcubierre's metric contains the combination $f v_{s}$ depending only on time, since $v_{s}=$ $\dot{x}_{s}, r_{s}(t), f\left(r_{s}\right)$. Thus, Christoffel's symbols are independent of spatial coordinates.

Equations (18) and (19) reduce to

$$
\begin{gathered}
-\frac{2 v_{s} \dot{v}_{s} f^{2}}{c^{3}} \frac{1}{1-\frac{\left(v-f v_{s}\right)^{2}}{c^{2}}}+\frac{\dot{v}_{s} f}{c^{2}} \frac{v^{2}}{c^{2}\left[1-\frac{\left(v-f v_{s}\right)^{2}}{c^{2}}\right]} \\
+\frac{1}{\sqrt{1-\frac{\left(v-f v_{s}\right)^{2}}{c^{2}}}} \frac{d}{d t} \frac{1}{\sqrt{1-\frac{\left(v-f v_{s}\right)^{2}}{c^{2}}}=0} \\
\frac{\dot{v}_{s} f}{c^{2}} \frac{\frac{2 v_{s}^{2}}{c^{2}}-1}{1-\frac{\left(v-f v_{s}\right)^{2}}{c^{2}}}+\frac{1}{c^{2} \sqrt{1-\frac{\left(v-f v_{s}\right)^{2}}{c^{2}}}} \frac{d}{d t} \frac{v}{\sqrt{1-\frac{\left(v-f v_{s}\right)^{2}}{c^{2}}}}=0,
\end{gathered}
$$

For $\dot{v}_{0}=0$ and $\dot{v}_{s} \neq 0$ these equations have solutions:

$$
\begin{aligned}
& v_{s}=\frac{v}{2 f}\left(\sqrt{1+\frac{2 v}{c}}-1\right), \\
& v_{0}=\frac{v}{2}\left(3-\sqrt{1+\frac{2 v}{c}}\right) .
\end{aligned}
$$

Hence $v \leq 4 c, v_{s} \leq \frac{v}{f}$ and $v_{0} \leq \frac{4 c}{3 \sqrt{3}}$. The local velocity is always below the speed of light. 
In case $v=v_{s}, r_{s}=\rho=$ const, hence the assumption $f=$ const is valid, formulae (25) and (26) take the form

$$
\begin{gathered}
v_{s}=2 c f(1+f), \\
v_{0}=2 c f\left(1-f^{2}\right),
\end{gathered}
$$

where $v_{0}<\frac{4 c}{3 \sqrt{3}}$ (i.e. the same as in the general case) and $v_{s}<\frac{4 c}{\sqrt{3}}$ obtained from (11) at $r_{s}=\rho$ valid for $x=x_{s}$. Thus, the starship velocity being equal to the deformation rate may exceed the speed of light, whereas the local velocity is always below it.

In case $r_{s} \gg R$ from (4) we have

$$
f=e^{-2 \sigma r_{s}} .
$$

For $\sigma r_{s} \gg 1$ the assumption $f=$ const is also valid, since the derivatives with respect to time enter into the geodesic only in the combination $\dot{f} v_{s}+f \dot{v}_{s}$, where the first term is negligible, with numerical calculations being unnecessary.

Then $v_{s}=4 c e^{2 \sigma r_{s}}$ at $v=4 c$. From (11) we have $\frac{4 c r_{s}}{\sqrt{3} \rho}>v_{s} \gg 4 e^{6} c$. Hence $r_{s} \gg e^{6} \sqrt{3} \rho$ and $\left|x-x_{s}\right| \gg \rho$.

Thus, the starship may much exceed the speed of light at the front of space-time deformation. $^{4}$

\section{Hawking Effect}

The energy of Hawking's radiation quanta

$$
\hbar \omega_{H}=2.8822 k T_{H},
$$

where

$$
k T_{H}=\frac{\hbar c}{2 \pi r_{g}}
$$

where $r_{g}$ is given by formula (8)

Using the formulae

$$
v=v_{s}, \quad r_{s}=\rho, \quad v_{s}>c, \quad \sigma R>8 \cdot 3^{1 / 4}\left(\frac{c r_{s}}{v_{s} \rho}\right)^{3 / 2},
$$

we obtain an estimate

$$
k T_{H}<\frac{\hbar c}{\pi \sqrt[4]{3} R} .
$$

The power of Hawking's radiation

$$
P_{H}=\frac{\hbar c^{6}}{15360 \pi G^{2} M^{2}} .
$$

For $R>100 \mathrm{~m}$ and $10^{-2} M_{\odot}$ we have $\hbar \omega_{H}<10^{-9} \mathrm{eV}$ and $P_{H}<10^{-15} \mathrm{erg} \mathrm{s}^{-1}$. Thus, Hawking's radiation does not affect the starship interior considerably. 


\section{Conclusion}

The possibility of a negative energy is evidenced by Casimir's effect used in electron microscopy, with the so-called quantum inequalities ${ }^{5}$ being negligible in perfect analogy to Schrödinger's cat paradox for macroscopic systems. ${ }^{6}$

The geodesic equations are solvable analytically in the one-dimensional case being natural for the starship motion along $\mathrm{x}$-axis. Thus, taking account of the derivative with respect to spatial coordinates is superfluous.

The difficulties related to a practical realization of the hypermotion lie in a too large amount of energy being required. However, we are dealing only with a conceptual possibility of hypermotion. Even in this case, there arise obstacles seeming insuperable nowadays. The conclusions based on the level of modern science are not ultimate, So much is unknown. Contrary to electromagnetism, we cannot control over gravity. When our knowledge becomes more detailed and extensive, it will be possible for us to succeed in revising many problems of gravity including its modern interpretation in the framework of general relativity.

\section{Acknowledgments}

We are grateful to the participants of the Ninth Alexander Friedmann International Seminar on Gravitation and Cosmology for useful discussion.

\section{References}

1. M. Alcubierre, Class. Quant. Grav. 11, L73 (1994).

2. F. S. Lobo and M. Visser, Class. Quant. Grav. 21, 5871 (2004).

3. B. McMonigal et al., Phys. Rev. D 85, 064024 (2012).

4. S. V. Krasnikov, Phys. Rev. D 57, 4760 (1998).

5. M. J. Pfenning and L. H. Ford, Class. Quant. Grav. 14, 1743 (1997).

6. E. Schrödinger, Naturwissenschaften B 23, 807 (1935). 